URSI GASS 2020, Rome, Italy; 29 Aug. - 5 Sep. 2020

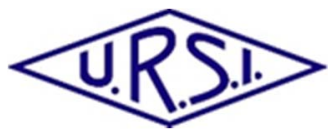

\title{
A Study of Antipodal Vivaldi Antenna for Microwave Imaging of Thermal Ablation
}

\author{
Mengchu Wang ${ }^{(1,2)}$, Lorenzo Crocco ${ }^{(1)}$, Marta Cavagnaro ${ }^{(1,2)}$ \\ (1) CNR-IREA National Research Council of Italy, Institute for Electromagnetic Sensing of Environment, Napoli, Italy, \\ wang.m@irea.cnr.it, crocco.1@irea.cnr.it \\ (2) DIET, Sapienza University of Rome, Rome, Italy, marta.cavagnaro@uniroma1.it
}

\begin{abstract}
This paper presents a study on the convergence criteria of a method of moment based software. A mesh convergence study on a simple dipole antenna and a more complex antipodal Vivaldi antenna was performed through WIPL-D environment. The Vivaldi antenna can be used for microwave imaging of liver thermal ablation treatments.
\end{abstract}

\section{Introduction}

Microwave imaging (MWI) is an imaging modality which can reconstruct the shape and dielectric properties of an unknown target based on measures of a scattered electromagnetic field. This imaging modality has been investigated by researchers all over the world in recent decades because of its potential for clinical use [1] [2]. Microwave imaging systems consist of several antennas that transmit and receive electromagnetic signals. The received signal is used to reconstruct the dielectric map of the target solving an inverse scattering problem. Antennas, as the most crucial part of the microwave imaging system, determine the system performances. In many existing medical imaging systems, ultra-wideband (UWB) antennas are used as the physical sensors, because they allow using frequency diversity in the algorithms [3].

A previous numerical analysis on the human abdominal region showed that a working bandwidth from 0.5 to $2 \mathrm{GHz}$ is a promising option for MWI because of good penetration of the electromagnetic signal into the human body tissues, and resolution potential [4]. In the same study, a matching medium with permittivity value of 23 was proposed to efficiently couple the electromagnetic (EM) signal emitted by the antennas to the human abdomen region, and also reduce the wavelength as compared to free space, being the relevant wavelength $\lambda / \sqrt{23}$.
The limited space of the human abdominal region on which locating the microwave imaging array requires low profile, compact, end-fire antennas. Vivaldi antennas are suitable candidates which meet all the above mentioned requirements. Many variations of the Vivaldi antennas exist, such as conventional Vivaldi antenna [5], antipodal Vivaldi antenna (AVA) [6], and balanced antipodal Vivaldi antenna (BAVA) [7]. Above all, the antipodal Vivaldi antennas (AVA) are the preferable choice for a MWI array thanks to their wide bandwidth, symmetric radiation patterns, and ease of fabrication. However, numerical design of these antennas could be challenging due to the fine details of the microstrip antipodal patches.

In this contribution, a mesh convergence study on the mesh of a method of moment based software (WIPL-D, d.o.o., Serbia) was performed. First, a simple dipole antenna was considered; then an antipodal Vivaldi antenna, which can be used for microwave imaging of thermal ablation treatments, was studied also.

\section{Methodology}

\subsection{WIPL-D Electromagnetic Simulation Software}

WIPL-D Pro CAD software environment (WIPL-D d.o.o., Serbia) was used in the numerical study [8]. WIPL-D Pro CAD is a software package for electromagnetic simulation of arbitrary 3D structures, which is based on the Methods of Moments (MoM) algorithm. MoM is one of the most widely used numerical techniques in computational electromagnetic. In $\mathrm{MoM}$, the radiating structure is segmented into surface patches in which equivalent currents, i.e. surface currents, are calculated. Then the Green function is used to evaluate the radiated electromagnetic field. MoM is an accurate computational method for metallic structures, in particular when the background medium is homogeneous and few scattering objects are present in the computation environment [9]. 


\subsection{Mesh Convergence Study}

A mesh convergence study was performed in the WIPL-D software environment to verify computational accuracy. In principle, the mesh model is first created using the fewest possible number of elements, and analyzed. Then the mesh density of the model is increased, and the simulation is redone looking for differences in the achieved results. This process is repeated until the results converge satisfactorily.

Two meshing techniques are present in the WIPL-D software: auto mesh and direct mesh. When using the auto mesh tool, the software segments all structures into small mesh elements. This mesh method is adaptive according to the structure shape. In particular, it allows coarser mesh elements for homogenously flat surfaces, and finer mesh elements for smaller detailed structures. When using this tool, the user defines a reference frequency, which limits the largest mesh element to 2 wavelengths by 2 wavelengths. The higher the reference frequency is, the finer the mesh will be, and the number of the unknowns increases accordingly. When using the direct technique, structures are directly meshed into quads of similar sizes, and the largest dimension is defined by the user. Usually, $\lambda / 10$ is the average mesh dimension to guarantee sufficiently accurate results, where $\lambda$ is the wavelength of the highest working frequency [11].

\subsection{Antenna models}

To perform the convergence study, a half-wavelength dipole antenna and an antipodal Vivaldi antenna were simulated into the software with different meshing techniques.

The dipole antenna was designed to work at $1.5 \mathrm{GHz}$ in air. Its total length is $100 \mathrm{~mm}$ and the wire diameter is $1 \mathrm{~mm}$. The mesh size for the direct mesh technique was chosen as $\frac{\lambda}{10}=20 \mathrm{~mm}$.

The Vivaldi antenna was designed following the steps proposed in [10], and considering it immersed into a matching liquid of relative permittivity $\varepsilon_{m m}$ equal to 23 . The antenna parameters were calculated according to the following equation:

$$
w=l=\frac{c}{f_{1}} \sqrt{\frac{2}{\varepsilon_{r}+\varepsilon_{m m}}}
$$

Where $\mathrm{w}$ is the antenna width, 1 is the antenna length, $\mathrm{c}$ is the speed of light in vacuum, $f_{1}$ is the lowest operation frequency, i.e., $0.5 \mathrm{GHz} . \varepsilon_{r}$ is the dielectric constant of the substrate of the antenna, which in this case, is RT6010 with an $\varepsilon_{r}$ value of 10.4 . The ground plane was redesigned to achieve the best $\mathrm{S}_{11}$ performances in the operating band. Then the optimizer was used to reduce the antenna width for a more compact design. A piece of the substrate between the signal layer and the ground layer was removed to improve the antenna radiation pattern at the higher frequencies. Figure 1 shows the Vivaldi antenna simulated in WIPL-D environment. On the left of the figure there is the front view, showing the antenna signal layer, and on the right there is the back view, showing the antenna ground layer. In the figure, the red shape represents the substrate part, and the light blue the metallic part.
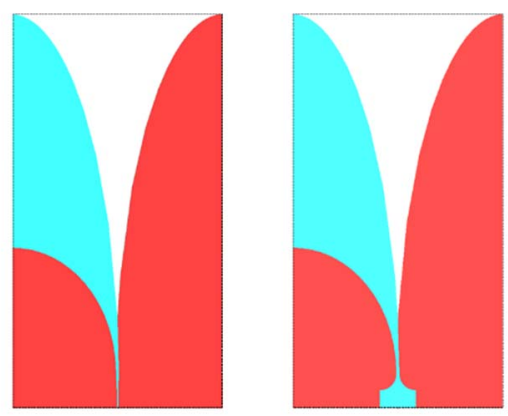

Figure 1. The Vivaldi antenna in WIPL-D environment (red: substrate, light blue: metal)

\section{Results and Discussion}

The convergence study was performed comparing the results, in terms of antenna matching, achieved using both the auto mesh technique, with different reference frequencies and the direct mesh one. The simulations were carried out with the antennas mentioned in the previous section. The relation of different meshing settings and numbers of unknowns is revealed in Table 1 .

Table 1. Mesh Settings and Corresponding Number of Unknowns

\begin{tabular}{|l|l|l|l|l|l|l|}
\hline & $\begin{array}{l}\text { Meshing } \\
\text { technique }\end{array}$ & \multicolumn{2}{|l|}{ Auto mode } & $\begin{array}{l}\text { Direct } \\
\text { technique } \\
\text { (Mesh } \\
\text { size } \lambda / \\
\mathbf{1 0}\end{array}$ \\
\hline Dipole & \begin{tabular}{l}
\multicolumn{4}{l}{} \\
Reference \\
frequency(GHz)
\end{tabular} & 0.5 & 1.5 & 3 & 7.5 & N/A \\
\cline { 2 - 7 } & $\begin{array}{l}\text { Number of } \\
\text { unknown }\end{array}$ & 1 & 3 & 5 & 9 & 5 \\
\hline $\begin{array}{l}\text { Vivaldi } \\
\text { antenna }\end{array}$ & $\begin{array}{l}\text { Reference } \\
\text { frequency(GHz) }\end{array}$ & 5 & 11 & 15 & 17 & N/A \\
\cline { 2 - 7 } & $\begin{array}{l}\text { Number of } \\
\text { unknown }\end{array}$ & 12438 & 36173 & 58789 & 73922 & 29656 \\
\hline
\end{tabular}

It is obvious that by increasing the reference frequency, the number of unknowns increases accordingly. For complex structures with more design details, like the Vivaldi antenna, a high number of unknowns are obtained by the meshing algorithms.

Figure 2 shows the reflection coefficient $\left(\mathrm{S}_{11}-\mathrm{dB}\right)$ of the half-wavelength dipole. From the figure it can be seen that for 
such simple structure, results converge with a few numbers of unknowns both by using the direct mesh technique, and by using the auto mode technique setting the reference frequency to the same value as the antenna resonance frequency.

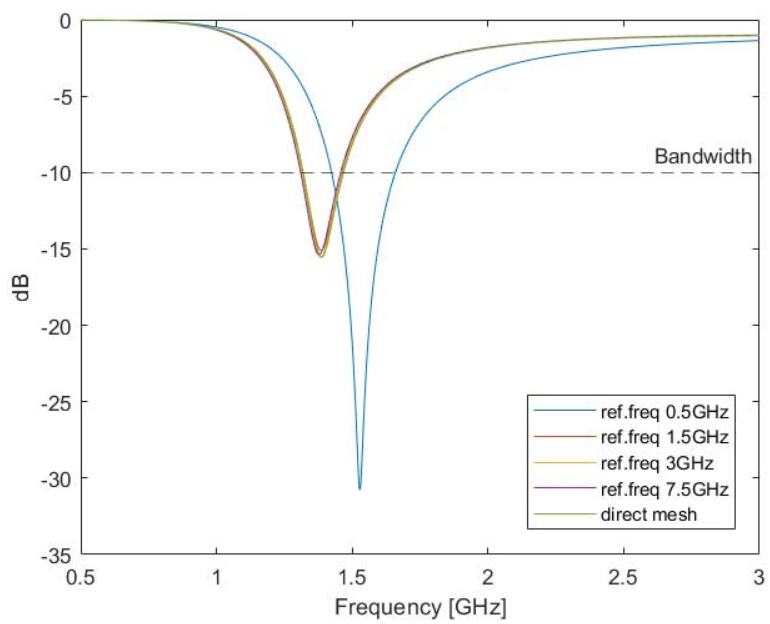

Figure 2. $\mathrm{S}_{11}(\mathrm{~dB})$ of a half-wavelength dipole

The second convergence study was performed on the Vivaldi antenna. The mesh size for the direct mesh technique at a working frequency of $2.5 \mathrm{GHz}$ is:

$$
\frac{\lambda}{10 \sqrt{\varepsilon_{m m}}}=2.5 \mathrm{~mm}
$$

The $\mathrm{S}_{11}$ results are shown in Figure 3.

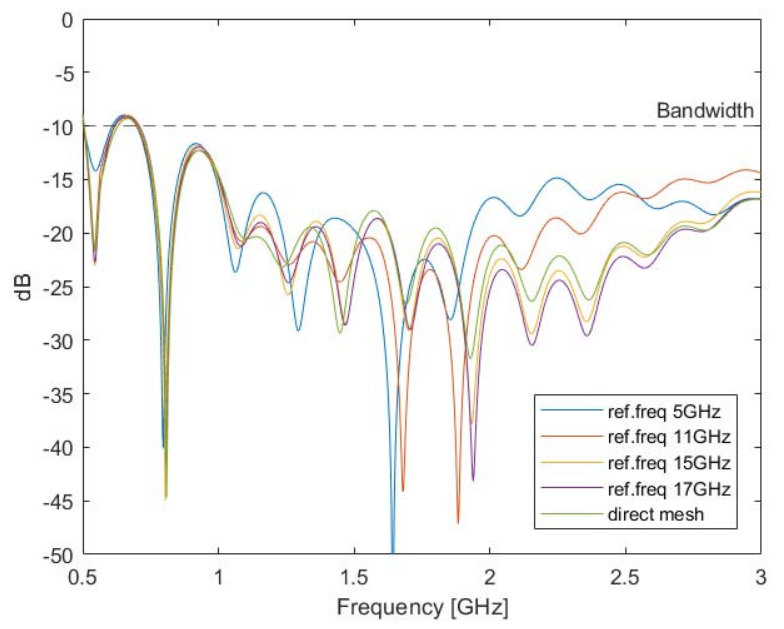

Figure 3. $S_{11}$ of the Vivaldi Antenna

From Figure 3, it is found that for such a complex structure with more design details than the dipole antenna, the $S_{11}$ varies a lot when different meshing techniques are used. In particular, when implementing the auto mesh technique, the results convergence satisfactorily only when the reference frequency is above 15 GHz. While using the direct mesh technique, the results converge even with a lower number of unknowns as compared with the auto mesh technique with a reference frequency of 11 GHz. From the reported results, it can be concluded that for complex structures it is preferable to choose the direct mesh technique.

\section{Acknowledgements}

This work was supported by the EMERALD project funded from the European Union's Horizon 2020 research and innovation program under the Marie Skłodowska-Curie grant agreement No. 764479.

\section{Reference}

[1] P. M. Meaney, P. A. Kaufman, L. S. Muffly, M. Click, S. P. Poplack, W. A. Wells, and S. D. Geimer, "Microwave imaging for neoadjuvant chemotherapy monitoring: initial clinical experience," Breast Cancer Research, 15, 2, 2013, R35.

[2] D. O’Loughlin, M. O’Halloran, B. M. Moloney, M. Glavin, , E. Jones, and M. A. Elahi, "Microwave breast imaging: Clinical advances and remaining challenges," IEEE Transactions on Biomedical Engineering, 65, 11, 2018, 2580-2590.

[3] O. M. Bucci, L. Crocco, T. Isernia, and V. Pascazio, "Inverse scattering problems with multifrequency data: reconstruction capabilities and solution strategies," IEEE Transactions on Geoscience and Remote Sensing, 38, 4, 2000, 1749-1756.

[4] M. Wang, M. Cavagnaro, and L. Crocco, "Initial Guidelines to the Design of a Microwave Imaging System for Ablation Monitoring," 14th European Conference on Antennas and Propagation (EuCAP), 2020 (Accepted).

[5] C. Deng, and Y. J. Xie, "Design of resistive loading Vivaldi antenna,". IEEE Antennas and Wireless Propagation Letters, 8, 2009, 240-243.

[6] A. Z. Hood, T. Karacolak, and E. Topsakal, A small antipodal Vivaldi antenna for ultrawide-band applications. IEEE Antennas and Wireless propagation letters, 7, 2008, 656-660.K.

[7] J. Bourqui, M. Okoniewski, , \& E. C. Fear, "Balanced antipodal Vivaldi antenna with dielectric director for near-field microwave imaging," IEEE Transactions on Antennas and Propagation, 58, 7, 2010, 2318-2326.

[8] B. M. Kolundzija, J. S. Ognjanovic, and T. Sarkar, "WIPL-D: Electromagnetic modeling of composite metallic and dielectric structures: Software and user's manual". Artech House, 2018.

[9] D. B. Davidson, Computational electromagnetics for RF and microwave engineering. Cambridge University Press, 2010.

[10] A. M. Abbosh, H. K. Kan, and M. E. Bialkowski, "Compact ultra wideband planar tapered slot antenna for use in a microwave imaging system,", Microwave and optical technology letters, 48, 11, 2006, 22122216.

[11] J, Richmond, "Scattering by a dielectric cylinder of arbitrary cross section shape," IEEE Transactions on Antennas and Propagation, 13, 3, 1965. 334-341. 